\title{
Scale in object and process ontologies
}

\author{
Femke Reitsma ${ }^{1}$ and Thomas Bittner ${ }^{2}$ \\ 1 \\ Department of Geography, 2181 LeFrak Hall, University of Maryland, College Park, \\ Maryland 20742, USA \\ femke@geog.umd.edu \\ 2 Institute for Formal Ontology and Medical Information Science, University of Leipzig, \\ Härtelstraße 16-18, 04107 Leipzig, Germany \\ thomas.bittner@ifomis.uni-leipzig.de
}

\begin{abstract}
Scale is of great importance to the analysis of real world phenomena, be they enduring objects or perduring processes. This paper presents a new perspective on the concept of scale by considering it within two complementary ontological views. The first, called SNAP, recognizes enduring entities or objects, the other, called SPAN, perduring entities or processes. Within the meta-theory provided by the complementary SNAP and SPAN ontologies, we apply different theories of formal ontology such as mereology and granular partitions, and ideas derived from hierarchy theory. These theories are applied to objects and processes and form the framework within which we present tentative definitions of scale, which are found to differ between the two ontologies.
\end{abstract}

Keywords. scale, granularity, hierarchy, process, ontology, mereology

\section{Introduction}

For geography as an empirical science, the observation of enduring entities and processes at different levels of granularity is of critical importance. Given a certain range of resolution only processes and enduring entities at certain levels of granularity can be observed. In geography this kind of problem is referred to as the problem of scale of observation, which has long been recognized as a central problem of the analysis of geographical phenomena. The concept of scale is controversial in its definition and often applied without clarification of its meaning (Lam and Quattrochi 1992). The meaning of scale discussed in this paper is not the traditional cartographic notion of scale, but rather the level at which reality is sampled and observed (Goodchild and Proctor 1997). Thus, scale is defined by and incorporates both grain and extent, spatial and temporal (Ahl and Allen 1996; Albrecht and Car 1999; Pereira 2002). 
In this paper we apply different theories of formal ontology such as mereology (Simons 1987) and granular partitions (Bittner and Smith 2001; Smith and Brogaard 2002), as well as hierarchy theory (Allen and Starr 1982, Ahl and Allen 1996), within the overarching framework of spatio-temporal ontology (Bittner and Smith 2003) in the context of considering the problem of scale. Our objective is to determine whether the combination of these theories provides new insights into the issue of scale. The aspect of scale that is focused upon is the linkage between scales, that is, the relationship between objects operating at large scales and small scales, and the relationship between processes operating at large scales and small scales. We consider a definition of scale for both enduring entities, such as humans and mountains, and processes, such as tidal currents and gentrification, within their formalization in different kinds of ontologies (SNAP-ontologies for enduring entities and SPAN-ontologies for processes).

The paper is structured as follows, Section 2 describes the notions of grain and extent and their use in the paper. Section 3 introduces the SPAN and SNAP ontologies as formal ways of representing objects and processes. Section 4 clarifies the notion of scale with regards to objects in a SNAP ontology, and Section 5 explores scale with regards to processes in a SPAN ontology. Section 6 concludes the paper.

\section{Grain and Extent}

Extent is concerned with the spatial size of phenomena, in $(x, y, z)$ dimensions, or the temporal length of duration over which those phenomena operate (Lam and Quattrochi 1992). For example, continental glaciers operate over a much larger extent, both spatial and temporal, than thunderstorms. Both spatial and temporal extent are referenced to an absolute space and time for the purpose of this paper. Grain, whether spatial or temporal, is a relative notion. That is, it has no absolute spatial or temporal location in the sense of being positioned on the earth at a certain longitude or latitude, or at a certain Greenwich Mean Time. Such positioning is defined by extent.

Grain refers to the fineness of distinctions recorded in the data, often referred to as resolution (Albrecht and Car 1999). For example, the spatial grain of a remotely sensed image is the size of each pixel in its relation to the patch of the earth it represents. Determining which spatial grain is appropriate for a certain observation is dependent on the spatial extent of the objects to be observed, that is, the spatial grain of our observation depends upon the granular structure of reality. Temporal grain refers to the frequency of behavior. For example, the temporal grain of a longitudinal study of the daily patterns of human movement is the frequency of observation or sampling. Determining which temporal grain is appropriate for a certain observation is dependent on the temporal extent of the objects to be observed, that is, the temporal grain of our observation depends upon the granular structure of reality in the sense that only a certain range of temporal grain is appropriate for the observation of temporal objects of a certain temporal extent. Frequency is traditionally defined as 
the number of cycles a phenomenon completes within a specified time interval. In the context of this paper, fast behavior is defined by high frequency and slow behavior by low frequency (Ahl and Allen 1996). For example, the movement of a glacier occurs at a much lower frequency than the ephemeral cusp formation at a beach.

The determination of the appropriate scale of analysis is fundamental to the analysis of all geographic phenomena. It is typically dependent on the objectives and resources of the research at hand. The effects of the scale of observation on geographic phenomena are well recognized and pertain to a common recognition that different processes are observed to operate at different scales, thus phenomena or processes may be obscured when research is concentrated at an inappropriate scale (Meyer et al. 1992). For example, an exclusive analysis of microclimates may lead to explanation based on local processes when there are processes of large extent, such as El Niño, that are influencing or controlling the microclimate and should be observed with coarser granularity. Although the problem of scale definition is well recognized, it continues as an unsolved problem, thus it remains a topical issue. Many questions continue unresolved from more recent initiatives such as NCGIA's (National Center for Geographic Information Analysis) Varanius project: Scale and Detail in the Cognition of Geographic Information, and the UCGIS (University Consortium for Geographic Information Science) research priority of scale (UCGIS 1998).

\section{Two Ontological Views}

Following Bittner and Smith (2003) we recognize two complementary views of the world, one directed towards enduring entities, or objects, and the other directed towards perduring entities, or processes. Taken together these two kinds of ontologies capture both enduring entities, such as political boundaries and humans, and spatio-temporal processes, such as erosion and urban sprawl. The former view gives raise to a series of snapshot-like ontologies called SNAP, each representing objects existing at a certain moment in time. The latter view presents an ontology called SPAN, which represents processes and other spatio-temporal entities as fourdimensional worms.

To describe geographic processes in an appropriate and complete manner we cannot have a SPAN ontology without the SNAP ontology and vice versa. For example, the processes of migration and gentrification cannot be understood without the enduring entities of humans, which are involved in these processes. Likewise, we cannot understand the interaction of sedimentary particles in the littoral zone of a coast without understanding processes such as longshore drift, rip currents, and mass transport. Thus the interaction between processes, enduring entities, and changes in enduring entities - which is related to participation in certain processes - is critical.

SNAP-ontologies are an intuitive approach to the world that is reflected in how we experience reality at every given moment in time, and corresponds to the way we 
typically model it: as a collection of interacting enduring entities and the relations between them. The temporal character of the world is reflected by a series of threedimensional temporal slices or snap-shots of enduring entities. Considering sequences of 3D-SNAPshots is the common approach to modeling processes in Geographic Information Systems (GIS); for example, in trying to model the demographics of a region over time we use snapshots of the state of the region at specified time intervals such as with census data. An important aspect is that processes are not represented in ontologies of the SNAP kind, although they can be discovered indirectly in terms of the changes they cause. Change is hereby defined as the difference in some attribute in an enduring entity at different time indexes, be that spatial location or some other property of the entity.

SPAN-ontologies are derived from four-dimensionalism, a popular position in contemporary analytic metaphysics (see Sider 2001). The four-dimensionalist views the world from a God's eye perspective, as spread out in time and populated by spacetime worms that represent processes. Time is considered as just another dimension in addition to the three spatial dimensions, as in the theory of relativity. Hence this is an atemporal view where things that are spread out over time are said to perdure. This does not deny the temporal extension of spatio-temporal entities, rather that their traditional temporal qualifiers of past, present, and future are traced over because all are simultaneously evident.

Another important aspect is that SPAN ontologies trace over enduring entities. For example, a flood event described by a SPAN ontology traces over the existence of enduring entities such as water bodies. However there exist complex interrelationships between SNAP and SPAN ontologies. Enduring entities at every moment in time have a corresponding three dimensional slice of the processes they participate in. For example, enduring entities such as humans or animals participate in migration processes. One cannot reduce one view to the other. Rather one must translate between SNAP and SPAN ontologies, requiring an analysis of crossontological relationships between those different ontological views on a metatheoretical level in order to establish a relationship between them, which will not be discussed in this paper (see Bittner and Smith 2003 for details). This is evident in the difficulties that GIS have with temporal phenomena because the process view of the world cannot be reconstructed with the view of enduring entities without losing something.

Within each ontology, scale is defined differently. We give a provisional definition of scale within the SNAP ontology, in terms of classes of objects. Here scale is a set of levels of granularity composed of objects that can be distinguished at these levels of granularity with observation or measurement within a certain range of spatial grain. 


\section{SNAP Objects, Granularity, and Scale}

We begin with a discussion of the granular structure of enduring entities, which is developed by applying the theories of mereology and granular partitions. Within this context the notion of scale is considered. The presented notions will then be extended to Section 5 in order to take into account the more complex granular structure of processes.

\subsection{Mereology and granularity trees}

Our basic tool for understanding the phenomenon of granularity is mereology (Simons, 1987). The most basic mereological concept is the relation of part to whole. The concept of part and whole applies in every domain, from ordinary objects such as people, chairs, and mangos, to processes such as frontal systems, information flows, and erosion. They may also apply to abstract entities such as classes and properties. In this section only enduring entities will be considered.

The simplest expression of this part-whole relation is given by $\leq$, where $x \leq y$ is read as ' $x$ is a part of $y$ '. For example, if a house (an enduring entity) is considered

a whole, its doors, roof, and windows are parts of that whole. The relation $\leq$ includes both the case of proper parthood $(<)$ and equality. The core axioms define the partwhole relation as reflexive, transitive, and antisymmetric, i.e., as a partial ordering (Simons 1987). In the context of this paper we are not interested in mereology in its whole generality but in hierarchical structures which represent mereological structure in a restricted form: as finite trees. Those structures are subject to the features of granularity.

Formally we define a granularity tree as a pair, $G=(R, \subseteq)$, where $R$ is a set of objects with a binary relation $\subseteq$ which is a restricted form of the mereological part-of relation. Following Smith and Brogaard (2002), we call the objects forming a system of granularities, cells, and the relation $\subseteq$, the subcell relation. Using the subcell relation we define the relations of overlap, proper subcell, and immediate proper subcell.

$$
\begin{array}{ll}
\text { D1 } & \text { O xy } \equiv \exists z(z \subseteq x \wedge z \subseteq y) \\
\text { D2 } & x \subset y \equiv x \subseteq y \wedge x \neq y \\
\text { D3 } & x \subset \text { I } y \equiv x \subset y \wedge \neg \exists z(x \subset z \wedge z \subset y)
\end{array}
$$

We here assume that quantification ranges over entities in $R$. Moreover we assume that leading quantifiers are understood. Two cells overlap if and only if they have a subcell in common (D1). The cell $\mathrm{x}$ is a proper subcell of the cell $\mathrm{y}$ if and only if $\mathrm{x}$ is a subcell of $y$ and $x$ and $y$ are not identical (D2). The cell $x$ is an immediate subcell of $\mathrm{y}$ if and only if $\mathrm{x}$ is a proper subcell of $\mathrm{y}$ and there does not exist a cell $\mathrm{z}$ such that $\mathrm{x}$ is a proper subcell of $\mathrm{z}$ and $\mathrm{z}$ is a proper subcell of $\mathrm{y}$. 
We continue by defining the predicates 'being a root element' and 'being an atom':

$$
\begin{array}{ll}
\text { D4 } & \text { Root } \mathrm{x} \equiv \forall \mathrm{y}(\mathrm{y} \subseteq \mathrm{x}) \\
\text { D5 } & \text { Atom } \mathrm{x} \equiv \neg \exists \mathrm{y}(\mathrm{y} \subset \mathrm{x})
\end{array}
$$

An entity is a root if and only if all entities are its subcells (D4). An entity is an atom if and only if it does not have a proper subcell.

Granularity trees are governed by the following axioms (Bittner and Smith (2003):

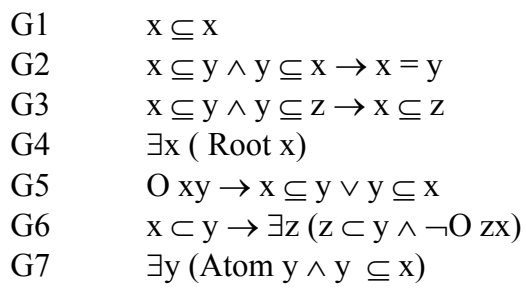

(G1-G3) ensure that the subcell relation is reflexive, antisymmetric, and transitive, i.e., a partial ordering. That there is a root element is guaranteed by (G4). Axiom (G5) ensures that if two cells overlap then one is a subcell of the other. This excludes partial overlap. For this reason there cannot be circles and the resulting structure is a tree. (G6) is known in the literature as the weak supplementation principle (Simons, 1987). It ensures that if a cell has a proper subcell then it has at least two nonoverlapping proper subcells. For this reason the resulting tree structure cannot degenerate into a list. (G7) ensures that every cell has an atom as subcell. Consequently, every cell is connected to the root cell by a finite chain of immediate subcells. This axiom might be disputable and is from a formal point of view not needed for the discussion that follows. However we believe that it is an important aspect of granularity that the subdivision into parts stops after finite steps.

Given those axioms one can prove that the root element is unique and that the strong supplementation principle holds, from which the extensionality of the overlap and the proper part relation follows (Simons, 1987).

It follows that every granularity tree can be represented using a tree structure in the mathematical sense, that is, as rooted directed graphs without circles, by taking the regions as nodes and by demanding that there is an edge from node $a$ to node $b$ if and only if $a \subseteq b$. For example, the parts of London may be referred to as 'boroughs', such as Westminster, Camden, Southwark, and Greenwich. The parts of these boroughs are referred to as 'suburb', for example, in the case of the borough of Camden, we find suburbs such as Kingcross, Hampstead Heath, Swiss Cottage, and Euston, which belong to such a system of granularities (Figure 1). Thus, we can describe London at varying levels of granularity, the composition of which defines our granularity tree. 
Granularity trees have finite depth, where depth is defined as the maximal length of the finite chain from the root to a leaf. Therefore the granularity tree can be described as having finite grain, that is, the range of grain (from largest to smallest) of a granularity tree is defined. Thus parts of the structured domain that are below a certain grain are not recognized in the tree in the sense that they are not cells of the tree connected to the root. For example, the parts of Euston are not recognized by the granularity tree in Figure 1. However, this granularity tree is not full, that is, its subcells do not exhaustively describe each cell (Bittner and Smith 2001). For example, London is composed of 32 boroughs in total.

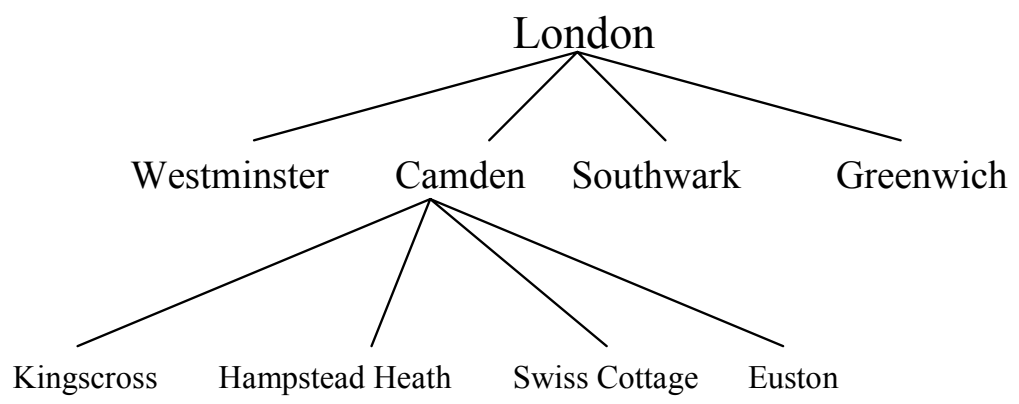

Fig. 1. London depicted as a granularity tree

Another example of granularity tree may be found in the classification of channel geomorphology (Montgomery and Buffington 1998). The watershed is the root of this granularity tree, which may be divided into sub-watersheds, sub-watersheds having valley segments as their parts, which are in turn composed of channel reaches, which are composed of channel units (Figure 2). 


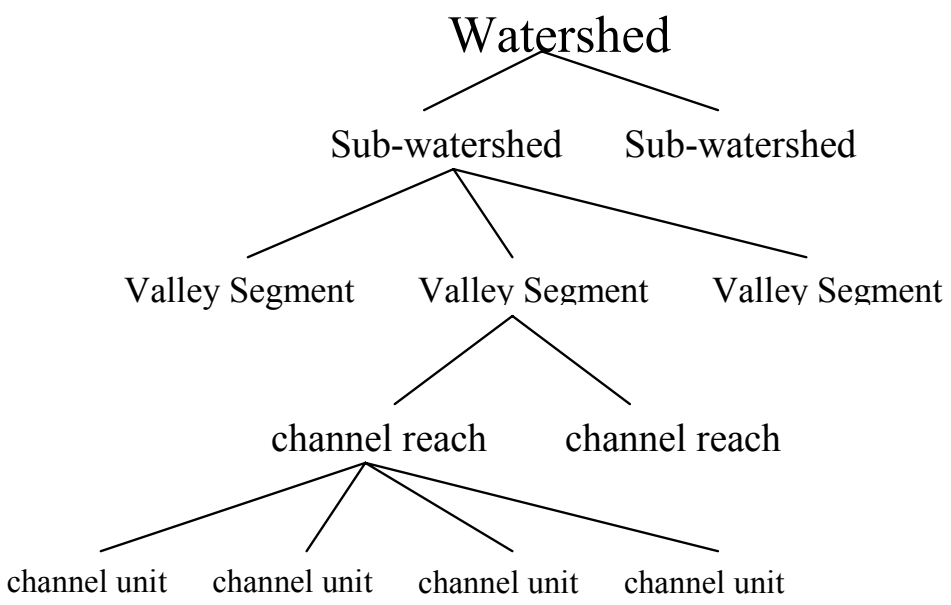

Fig. 2. Geomorphological granularity tree

Take for example the Chesapeake Bay Watershed, the sub-watersheds in this watershed include the Potomac River, Susquehanna, York, and James. In the Potomac River sub-watershed, valley segments may include the north and south fork of the Shenandoah river, Monocacy River, Anacostia River, and the lower Potomac River (Figure 3). This granularity tree could be expanded to include channel reaches and channel units.

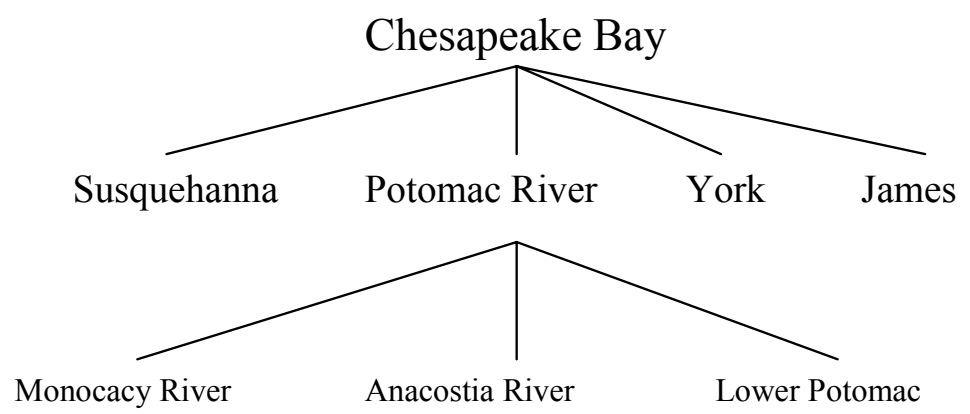


Fig. 3. Chesapeake Bay described as a granularity tree

The example of the Chesapeake Bay provides grounds for introducing the distinction between bona fide and fiat granularity trees. The objects within the granularity tree can be either bona fide or fiat (Smith 1995; Bittner and Smith 2001). Bona fide objects exist independently of human partitioning activity, take for example the Potomac River, which exists regardless of how it may be organized into a granularity tree. Fiat objects are created by our partitioning activity, for example the census units used to divide the population into spatial partitions. Orthogonal to this division between bona fide and fiat objects is the division of hierarchies into hierarchies of kinds (taxonomies) and hierarchies of parts (partonomies) (Tversky 1990). A taxonomy is the partitioning of reality into kinds, for example a poodle is a kind of dog, which is a kind of animal. A partonomy is the partitioning of reality into parts. The Geomorphologic granularity tree (Figure 2), instantiated by the Chesapeake Bay granularity tree (Figure 3 ), is an example of a partonomy.

\subsection{Levels of granularity}

Let $G=\left(R_{G}, \subseteq\right)$ be a granularity tree and let $R_{\mathrm{G}}$ be non-empty. Levels of granularity in $G$ are then defined as sets of cells inductively as follows (Rigaux and Scholl 1995):

1. The set containing only the root cell of the granularity tree is a level of granularity

2. Let $C$ be a level of granularity consisting of the cells $\mathrm{z}_{1}, \ldots, \mathrm{z}_{\mathrm{n}}$. Then we can replace every $z_{i}$ by its immediate subcells (if it has any) and the result is another level of granularity.

Consider Figure 1; the levels of granularity, for example, are:

$g_{0}\{$ London $\}$

$g_{1}\{$ Westminster, Camden, Southwark, Greenwich $\}$

$g_{2}\left\{\begin{array}{l}\text { Westminster, Kingscross, Hampstead Heath, Swiss Cottage, Euston, Southwark, } \\ \text { Greenwich }\end{array}\right\}$

Our definition captures only certain necessary conditions that characterize levels of granularity, which are purely mereological in nature:

1. The elements forming a level of granularity are pair-wise disjoint 
2. Levels of granularity are exhaustive in the sense that for every cell $z$ that does not belong to the granularity-level $\delta=\left(z_{1}, \ldots z_{k}\right)$ there exists a cell $z_{\mathrm{i}} \in \delta$ such that $\mathrm{z}_{\mathrm{i}} \subseteq \mathrm{z}$ or $\mathrm{z} \subseteq \mathrm{z}_{\mathrm{i}}$.

Our definition, so far is relatively rough, for example, the lengths of each finite chain that connect each leaf cell to the root cell in the granularity tree are not equal. This results in the problem of objects being repeated at a number of levels of granularity. In the London example, Westminster, Southwark, and Greenwich, are repeated in level $g_{2}$ after their initial appearance in level $g_{1}$.

For a more complete characterization of levels of granularity more domain-specific properties need to be taken into account. In SNAP domains metrical notions could also be included, for example, we might impose the requirement that objects forming a certain level of granularity have roughly the same size. In SPAN domains, as we shall see in Section 5 below, specific properties of the processes studied need to be considered.

\subsection{Resolution of observation and scale}

With the organization of objects into a granularity tree we return to the distinction between the granular structure of reality and the resolution of observation that was introduced in the introduction. Given a bona fide granularity tree, its ability to reflect the granular structure of reality that we wish to describe depends on the resolution of observation. Objects of human scale, such as books, filing cabinets, elephants, and anything else at the level of Zubin's A-spaces (Zubin 1989), are observable by humans without other instruments. Objects of larger scale, such as cities, watersheds, and islands, and of smaller scale, such as cells, bacteria, and atoms, require a different resolution of observation that depends on scale specific observation tools.

Scale, then, is determined by the relationship between the granular structure of reality and the resolution of observation. Returning to the provisional definition given at the end of Section 3, scale is a set of levels of granularity in the defined granularity tree that is partitioned into equivalence classes that are distinguished on the granular structure of reality through a certain range of resolution of observation. For example, human scale, as a class of levels of granularity, is composed of objects that can be distinguished on the granular structure of reality through a range of resolution defined by the limits of our perception.

The notion of scale discussed here only recognizes the relative extent and grain of objects based on their description as a granularity tree. The root cell in a system of cells is of greater relative scale than its children, as are any lower levels of granularity with respect to their descendents. Thus, going down the levels of granularity from the root cell, with coarsest relative granularity, to leaf-cells of finest relative granularity, the granularity tree is organized from phenomena of large relative extent to phenomena of small relative extent. For example, London is much larger than any of its descendents (Westminster, Camden, Southwark, Greenwich) taken individually. 
We may then define scale on our system of granularities as the partition of the set of levels of granularities into equivalence classes observable at a certain range of resolution, where from the root cell to depth $\mathrm{x}$ is scale 1 , from depth $\mathrm{x}$ to $\mathrm{y}$ is scale 2 . However, the definition of these depths is not specified in mereology, the observer defines them.

\section{SPAN Processes, Granularity, and Scale}

As with Section 4, we begin by discussing granular structures using the theories of mereology and granular partitions, however here we focus on perduring entities or processes. Within the framework provided by these theories of formal ontology applied to processes, the notion of scale is considered.

\subsection{Process mereology}

In geography we are typically interested in perduring entities, or processes, rather than in enduring entities, or objects. Spatio-temporal entities, or processes, are best described by a SPAN-ontology. We may also distinguish between processes and classes of processes. For example, tropical cyclones are a class of processes and tropical cyclone Paka is an instance of this class.

As with the mereology of enduring entities, the relations of parthood and proper parthood also apply to the processes, or perduring entities, of a SPAN-ontology. However, the mereological part-of relation behaves differently in SNAP and SPAN ontologies in the sense that enduring entities have only spatial parts. Perduring entities, in contrast, also have temporal parts. For example, a temporal part of a process such as gentrification may be the temporal interval over which rent is increased by a certain amount; another temporal part of that same process may the temporal interval over which inhabitants are evicted.

This means that in the domain of enduring entities, granularity is a spatial notion, whereas it is a spatio-temporal notion in the domain of processes. These processes have spatial and temporal extent and spatial and temporal grain, and are structured mereologicaly. Therefore their organization into a tree of granularity must consider both their spatial and temporal extent and their spatial and temporal grain.

\subsection{Hierarchy theory and processes}

In descriptions of the process class of urban growth we can move from the level of granularity defined by processes at the neighborhood level to that level defined by processes observed at the metropolitan statistical area level. Or, in the case of weather phenomena, we can describe them at the granularity of microclimates or large-scale phenomena such as the El Niño weather pattern. The part-whole nature of these processes defines the hierarchy, or granularity tree, that they compose (note that 
hierarchy and granularity tree are taken to be synonymous). The organization of processes into a hierarchy is developed in hierarchy theory. Hierarchy theory, propounded by Ahl and Allen (1996) and Allen and Star (1982), is based on the recognition that processes, spatio-temporal phenomena such as urban growth or weather phenomena, can be described at different levels of granularity.

There is a strong consonance between hierarchy theory, mereology, and granular partitions, at least at the conceptual level. Simon (1973), one of the foundational thinkers of hierarchy theory, uses some of the mereological basics and the tree-like systems of granularities, defining a hierarchy as a partial ordering, a tree. The basic mereological axioms, noted above in Section 3.1, hold true for hierarchy theory, however, hierarchy theory does not formalize these relationships between parts and wholes in any form of explicit calculus. Its novelty lies in its rules for the organization of these parts and wholes into a hierarchy of spatio-temporal processes. We interpret hierarchy theory as an extension to mereology-based notions of parthood, scale, and levels of granularity with the specification of the relationship between levels, thereby extending our definition of scale above. It defines a number of ordering principles of upper levels relative to lower levels of granularity for processes (Ahl and Allen 1996). In this paper we will focus on two of these principles, that is, higher levels in the hierarchy behave at lower frequency than lower levels, and, as with mereological structure, higher levels contain processes at lower levels as parts. Our granular structure is defined by both spatial and temporal grain and extent.

\subsection{Granularity trees formed by processes}

Processes have sub-processes as their parts. We organize processes and their parts into granularity trees using spatio-temporal grain and extent, extending beyond the granular structure of enduring entities by paying particular attention to temporal grain, or frequency. Frequency, as described in Section 2, is traditionally defined as the number of cycles a phenomenon completes within a specified time. Therefore, processes of high frequency are characterized by fast behavior where processes recur within a short duration of time, and processes of low frequency are characterized by slow behavior where processes recur within a long duration of time. For example, tides are a low frequency class of processes, oscillating over a 12 hour period, compared to the relative high frequency of a wave, which recurs over mere seconds.

Extending this notion of the frequency of a process hierarchically, each process is composed of a pattern of processes at a lower level of granularity, the parts of the process. A pattern of processes, then, is a sequence of different individual processes. The frequency of a process is the reoccurrence of the pattern of processes that it is composed of. Take, for example, a typhoon, which is a class of processes that have a certain lifecycle they undergo, which are characterized by a certain pattern of processes. The pattern of processes of a typhoon is likely to include a tropical depression and a tropical storm. The frequency of a typhoon is then the reoccurrence of the same pattern of processes. 
Processes of low frequency, which recur over a long duration of time, are higher in the granularity tree than processes of high frequency, which recur over a short duration of time. This relationship between process at a higher level in the granularity tree and patterns of individual processes at a lower level is repeated down through the granularity tree. For example the temporal grain or frequency of atmospheric phenomena are typically classified as micro-scale: seconds to minutes, meso-scale: minutes to days, synoptic scale: days to weeks, and macro-scale: weeks and greater (Ahrens 1991). Another example of a system of granularities is defined on coastal processes. Consider the tidal cycle in the Bay of Fundy as a granularity tree (Figure 4). Note that this is a class of processes that can be applied to any instance of a tidal cycle in the Bay of Fundy. One tidal cycle occurs over almost a 13-hour period, the parts of which are its currents which have a higher frequency, followed by the subprocesses of waves which have a higher frequency again (Figure 5). The difficulty with finding an appropriate geographic example is that few researchers describe processes hierarchically, thus the example given above is very incomplete and provisional.

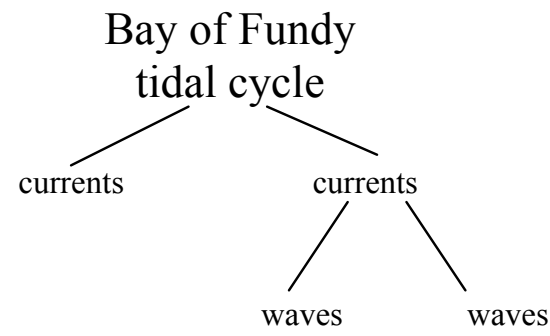

Fig. 4. The Bay of Fundy tidal cycle as a granularity tree 


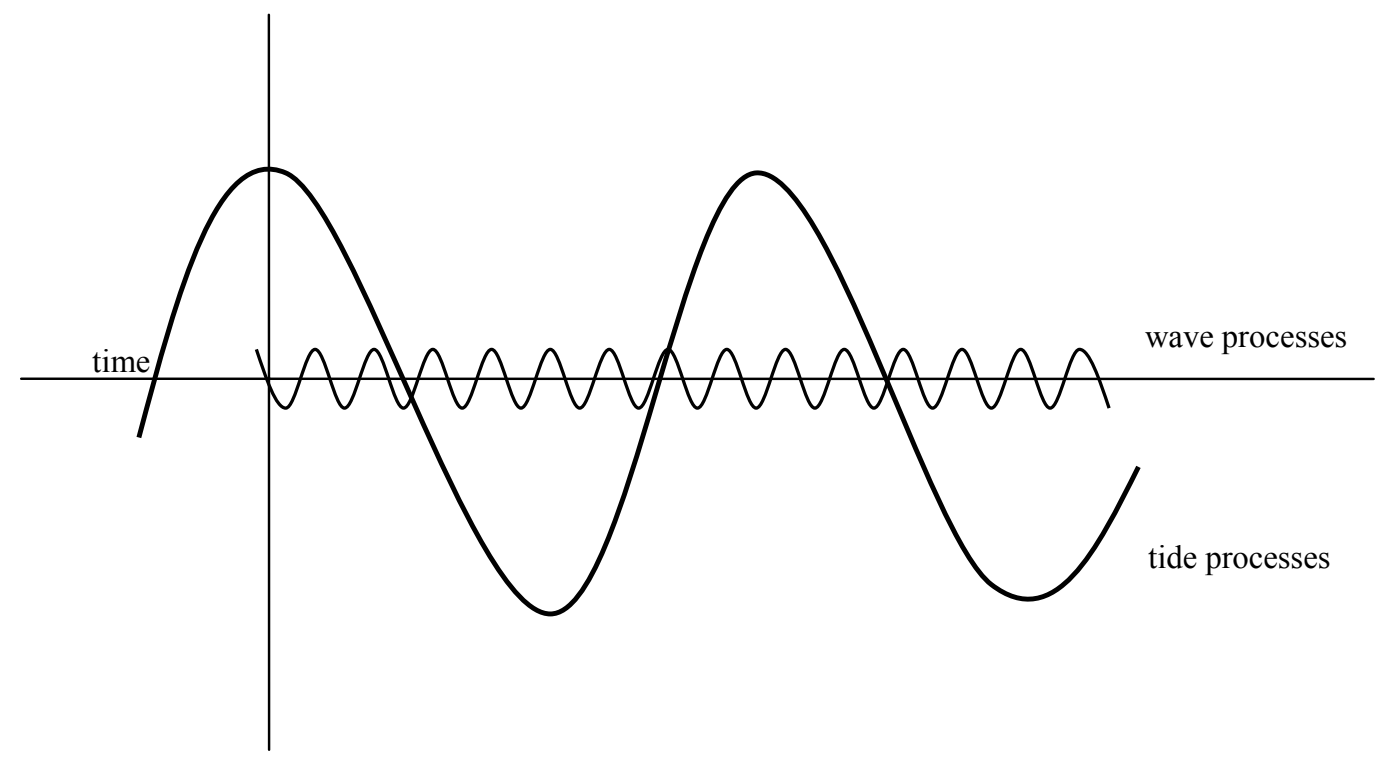

Fig. 5. A pattern of wave processes superimposed on the tide process

The temporal frequency of processes often reflects their spatial and temporal extent. Processes that are higher in our granularity tree are not only characterized by lower temporal frequency but also greater extent, thus processes that are lower in the system have higher frequency and smaller spatial and temporal extent. This reflects a well recognized principle whereby phenomena that are large in space, such as El Niño, have a low frequency, whereas small phenomena, such as micro-climates, have a higher frequency (Meyer et al. 1992). For example, take the classification of channel geomorphology, which incorporates both the structural geomorphology and the functional geomorphic processes operating at each level. The processes operating in a channel unit are contained, spatially and temporally, in those of a channel reach, which are contained within those processes operating in a valley segment, and so forth within a watershed and a geomorphic province (Montgomery and Buffington 1998). Similarly, in the Bay of Fundy example it can be said that the tidal cycle contains all of the lower processes in the granularity tree within its temporal and spatial extent.

A system of process granularities is a pair, $G_{p}=(P, \subseteq)$, where $P$ is a set of processes with a binary relation $\subseteq$ satisfying G1-G7 given above. As with enduring entities, we call the processes forming a system of granularities, cells, and the relation $\subseteq$, the subcell relation. However, for processes this subcell relation has to obey 
additional constraints which are defined in terms of spatial grain and extent and temporal grain and extent, where a subcell must:

1. have smaller temporal extent

2. have smaller spatial extent

3. have greater temporal frequency, or finer temporal grain

4. have greater spatial resolution, or finer spatial grain

\subsection{Levels of process granularity}

The nature of the part-whole relationship for processes, beyond the requirements of mereology, can then be summarized as: a part is contained within the spatial and temporal extent of the whole and has a higher frequency (temporal grain) and higher resolution (spatial grain) than the whole. In mereology, the parts exhaustively sum to the whole, such as the full granularity tree for London that would include all of its 32 Boroughs completely covering London's spatial extent. In contrast, the granularity tree that is formed by processes is not spatio-temporally exhaustive.

Within a granularity tree we can distinguish levels of granularity at which certain processes reside. Because of the added temporal dimensions of processes, our organization of processes into a granularity tree reflects this temporality. The sequence of processes that composes a process at a higher level has the requirement of having the same (or a similar range of) frequency or temporal granularity, which is the primary organizing construct of the granularity tree. Thus the levels in a granularity tree and the depth of each level is defined by the frequency of the process, that is, the recurrence of a sequence or pattern of sub-processes that compose a process. The approach for slicing a granularity tree into levels defined in Section 4.2 applies to processes also.

\subsection{Process, resolution of observation, and scale}

As with objects, in Section 4.3, our definition of a system of spatio-temporal granularity that reflects the granular structure of reality depends on the resolution of observation. Processes at different scales require different resolutions of observation. However, unlike objects, with processes this notion of resolution is both spatial and temporal, that is, both spatial and temporal extent and spatial and temporal grain must be considered. For example, processes of human scale are observable without other instruments, such as the cusp formation at a beach, or pedestrian traffic along a limited stretch of pavement. Larger scale processes, such as oceanic circulation and urban sprawl, and smaller scale processes such as cancer or the migration of dust mites, require a different resolution of observation that depends on spatial and temporal scale specific observation tools. From the perspective of a SPAN-ontology, the length of the space-time worm that describes our process defines the temporal extent and implies a certain temporal resolution of observation to capture the temporal grain of the process. Likewise, the three dimensional geometry of that space-time 
worm defines the spatial extent and therefore implies a certain spatial resolution of observation to capture the spatial grain of the process. As noted earlier, the spatial and temporal dimensions are independent. This inclusion of temporal resolution extends to the definition of scale for processes. For example, human scale processes, as a class of levels of spatio-temporal granularity, are composed of processes that can be distinguished on the granular structure of reality through a range of spatial and temporal resolution that is defined by the spatial and temporal limits of our perception.

Given the definition of scale in Section 4.4, it may be extended by considering the temporality of processes and by defining the relationship between levels of granularity as the frequency of the process under observation. We may then define scale on our system of granularities as the partition of the set of levels of granularities into equivalence classes of processes observable within a certain range of frequency or temporal granularity. From the root cell to depth $\mathrm{x}$ is scale 1, from depth $\mathrm{x}$ to $\mathrm{y}$ is scale 2 . Frequency classes determine the depths. More specifically, we may define a cut by the natural breaks in the observed frequency of a phenomena, where 'the natural breaking points in systems, the natural surfaces about which systems are nearly decomposed, have been identified as portions of the scale gradient which are so steep that they can be functionally considered as steps' (Allen and Star 1982). Alternatively, to define the depths of scale we might temporalise other classification measures such as Equal Interval or Standard Deviation.

\section{Conclusions}

Understanding the organization of processes into hierarchically structured granularity trees, where patterns of sub-processes at finer levels of granularity sum up to processes at coarser levels of granularity, helps us to understand the interrelationships between phenomena at different levels of granularity. These granularity trees form a framework for partitioning the world of enduring entities, viewed through the SNAP ontology, and processes, viewed through the SPAN ontology. Within both ontologies, the spatial and temporal grain of observation identifies the levels of the granularity tree. For enduring entities, their spatial extent and grain defines the structure of the granularity tree. For processes, their spatial and temporal grain and spatial and temporal extent defines the structure of the granularity tree. We defined scale as the depth between partitioned equivalence classes of either objects or processes described by the granularity tree.

It is, however, important to stress that our examples are somewhat simplified and completely ignore the issue of vagueness usually involved. This extends beyond defining the spatial bounds of objects to include the temporal dimension of defining the spatio-temporal bounds of processes. For example, what defines the beginning of a tropical cyclone? What are the pattern of processes that indicate the precise instant of a migration process? 
Other questions that surface from this research include the exploration of bona fide and fiat hierarchies and the relationship between them. Furthermore, consideration the influence of varying projections on our hierarchy, not only varying in the sense of different views on the same subject matter, but also varying dynamically where scales change over time, that is, we have dynamic granularity trees, or dynamic hierarchies. Furthermore, the difficulty in finding hierarchical descriptions of processes presents the question of whether or not the world is organized hierarchically or whether our tools and views are limited.

\section{Acknowledgements}

We would like to thank Jochen Albrecht for many helpful comments and valuable insights throughout the development of this paper, and support from the Wolfgang Paul Program of the Alexander von Humboldt Foundation is gratefully acknowledged.

\section{References}

Ahl, V. and T. F. H. Allen (1996). Hierarchy Theory: a vision, vocabulary, and epistemology. New York, Columbia University Press.

Ahrens, C. D. (1991). Meteorology Today: an introduction to weather, climate, and the environment. New York, West Publishing Company.

Albrecht, J. and A. Car (1999). GIS analysis for scale-sensitive environmental modelling based on hierarchy theory. In: GIS for Earth Surface Systems. R. Dikau and H. Saurer. Berlin, Gebruder Borntraeger: 1-23.

Allen, T. F. H. and T. B. Starr (1982). Hierarchy Theory: perspectives for ecological complexity. Chicago, The University of Chicago Press.

Bittner, T. and B. Smith (2001). A Taxonomy of Granular Partitions. COSIT 2001, Morro Bay, Lecture Notes in Computer Science, Berlin-Heidelberg, Springer-Verlag.

Bittner, T. and B. Smith (2003). Granular Spatio-Temporal Ontologies. AAAI Spring Symposium on Foundations and Applications of Spatio-Temporal Reasoning (FASTR), Palo Alto, California.

Goodchild, M. F. and J. Proctor (1997). Scale in a Digital Geographic World. Geographical \& Environmental Modelling 1(1): 5-23.

Lam, N. S. and D. A. Quattrochi (1992). On the Issue of Scale, Resolution, and Fractal Analysis in the Mapping Sciences. Professional Geographer 44(1): 88-98.

Meyer, W. B., D. Gregory, B. L. T. II and P. F. McDowell (1992). The Local-global Continuum. In: Geography's Inner Worlds. J. M. Olson. New Jersey, Rutgers University Press: 255-279. 
Montgomery, D. R. and J. M. Buffington (1998). Channel Processes, Classification, and Response. In: River Ecology and Management: Lessons from the Pacific Coastal Ecoregion. R. J. Naiman and R. E. Bilby. New York, Springer-Verlag.

Pereira, G. M. (2002). A Typology of Spatial and Temoral Scale Relations. Geographical Analysis 34(1): 21-33.

Rigaux, P. and M. Scholl (1995). Multi-scale partitions: Application to spatial and statistical databases. Advances in Spatial databases (SSD '95) - Lecture Notes in Computer Science, Berlin, Springer-Verlag.

Sider, T. (2001). Four-Dimensionalism. Oxford, Clarendon Press.

Simon, H. A. (1973). The Organization of Complex Systems. In: Hierarchy Theory: the challenge of complex systems. H. H. Pattee.

Simons, P. (1987). Parts: a study in ontology. Oxford, Clarendon Press.

Smith, B. (1995). On Drawing Lines on a Map. Spatial Information Theory, Proceedings of COSIT '95, Berlin, Springer Verlag.

Smith, B. and B. Brogaard (2002). Quantum Mereotopology. Annals of Mathematics and Artificial Intelligence 36(1-2): 153-175.

Tversky, B. (1990). Where Partonomies and Taxonomies Meet. In: Meanings and Prototypes: studies in linguistic catogorization. S. L. Tsohatzidis. London, Routledge.

UCGIS (1998). Scale. Research Priorities: revised white papers.

Zubin, D. (1989). Natural Language Understanding and Reference Frames. In: Languages of Spatial Relations: Initiative 2 Specialist Meeting Report Technical Paper 89-2. D. M. Mark, A. Frank, M. J. Egenhofer et al. Santa Barbara, CA, National Center for Geographic Information and Analysis: 13-16. 